\title{
VARIETIES OF COMBINATORIAL GEOMETRIES
}

BY

J. KAHN ${ }^{1}$ AND J. P. S. KUNG

\begin{abstract}
A hereditary class of (finite combinatorial) geometries is a collection of geometries which is closed under taking minors and direct sums. A sequence of universal models for a hereditary class $\mathcal{T}$ of geometries is a sequence $\left(T_{n}\right)$ of geometries in $\mathcal{T}$ with rank $T_{n}=n$, and satisfying the universal property: if $G$ is a geometry in $\mathcal{T}$ of rank $n$, then $G$ is a subgeometry of $T_{n}$. A variety of geometries is a hereditary class with a sequence of universal models.

We prove that, apart from two degenerate cases, the only varieties of combinatorial geometries are

(1) the variety of free geometries,

(2) the variety of geometries coordinatizable over a fixed finite field, and

(3) the variety of voltage-graphic geometries with voltages in a fixed finite group.
\end{abstract}

1. Introduction. The notion of free objects in a variety is one of the most important and pervasive ideas in universal algebra. In this paper, we investigate how that notion can be interpreted in the context of the theory of combinatorial geometries (or matroids). No detailed knowledge of universal algebra is required for reading this paper. However, we do assume familiarity with the basic concepts of the theory of combinatorial geometries [2, 4 and 13].

To fix our terminology, let $G$ be a finite geometric lattice. Its maximum and minimum are denoted by $\hat{1}$ and $\hat{0}$. Let $S$ be the set of points (or atoms) in $G$. The lattice structure of $G$ induces the structure of a combinatorial geometry, also denoted by $G$, on $S$. The cardinality $|G|$ of the geometry $G$ is the cardinality of the set $S$ of points. All geometries appearing in this paper are assumed to be of finite cardinality.

Let $T$ be a subset of $S$. The deletion of $T$ from $G$ is the geometry on the point set $S \backslash T$ obtained by restricting $G$ to the subset $S \backslash T$. The contraction $G / T$ of $G$ by $T$ is the geometry induced by the geometric lattice $[\bar{T}, \hat{1}]$ on the set $S^{\prime}$ of all flats in $G$ covering $\bar{T}$ (the closure of $T$ ). Thus, in our convention, the contraction of a geometry is always a geometry-indeed, it is isomorphic to the simplification of the matroid $G / T$ as it is usually defined. A geometry which can be obtained from $G$ by deletions and contractions is called a minor of $G$. Minors are the subobjects in the category of combinatorial geometries and strong maps.

Finally, we note that we shall often use "is" for "is isomorphic to" when precision threatens to turn into pedantry.

Received by the editors July 7, 1980.

AMS (MOS) subject classifications (1970). Primary 05B35, 08A15.

${ }^{1}$ Supported in part by an AMS Fellowship and ONR Contract \#N0014-76-C-0366.

(C)1982 American Mathematical Society 0002-9947/82/0000-1046/\$04.75 
2. Varieties of combinatorial geometries. A collection $T$ of combinatorial geometries is a hereditary class if it is closed under taking minors and direct sums. More precisely, a hereditary class $\mathcal{T}$ satisfies the following axioms.

H1. If $G$ is in $\mathcal{T}$ and $H$ is isomorphic to a minor of $G$, then $H$ is also in $\%$.

H2. If $G$ and $H$ are in $\mathcal{T}$, then so is their direct sum $G \oplus H$.

Hereditary classes of geometries have been discussed earlier, most notably in [9], but little is known about them.

Formally, the definition of a hereditary class is a direct analog of the definition of a variety in universal algebra (see, for example, [3]). However, in universal algebra, a variety $\mathcal{q}$ of algebras is a very structured collection of objects. For example, a theorem of Birkhoff says that it can be defined by equations. A consequence of this is the existence for each $n$ of a free algebra on $n$ generators - that is, there exists an algebra $F_{n}$ in $\tau$ defined by the universal property: if $A$ is an algebra in $T$ generated by $n$ elements, then $A$ is a subobject of $F_{n}$.

What is the analogue of free algebras for hereditary classes? A reasonable definition is the following. A sequence of universal models for a hereditary class $\widetilde{T}$ of geometries is a sequence $\left(T_{n}\right)$ of geometries such that

U1. $T_{n}$ is in $\mathcal{T}$ and is of rank $n$.

U2. If $G$ is a geometry of rank $n$ in $\mathcal{T}$, then $G$ is a subgeometry of $T_{n}$.

In contrast to the situation in universal algebra, sequences of universal models need not exist. Take for example, the hereditary class of all geometries, or the class of all totally unimodular geometries.

DEFINITION. A variety $T$ of combinatorial geometries is a hereditary class of geometries with a sequence of universal models.

There are two important examples of varieties. Let $\varrho(q)$ be the collection of all geometries coordinatizable over the finite field $G F(q)$. The projective geometries $P_{n}(q)$-here, $n$ is the rank of $P_{n}(q)$ as a geometric lattice-are universal models for $L^{2}(q)$. A less well-known example is the collection $\mathscr{Z}(A)$ of voltage-graphic geometries with voltages in a finite group $A$; the universal models here are the Dowling geometries $Q_{n}(A)$ (see [7]). These voltage-graphic geometries were discovered and studied by Zaslavsky $[\mathbf{1 4}, \mathbf{1 5}]$. We shall need only the basic properties of Dowling geometries and they will be recounted at the appropriate moment. We should mention that when $A$ is the trivial group, $Q_{n}(A)$ is the graphic geometry of the complete graph (on $n+1$ vertices) and the associated variety $\mathscr{Z}(A)$ is the variety of graphic geometries.

One is at this point hard put to think of additional examples. The reason for this is that, apart from degenerate cases, there are no others. This statement, which will be made precise in $\S 9$, is our main result. We proceed first to its proof.

3. Modular flats. Henceforth, $\left(T_{n}\right)$ is a sequence of universal models for a variety $T$ of geometries.

LEMMA 1. Let $G$ be a geometry in $T^{T}$ and let $t_{i}$ be a subgeometry of $G$ isomorphic to $T_{i}$. Then $t_{i}$ is a modular flat of $G$.

Proof. First observe that $t_{i}$ is of rank $i$ and its closure is a subgeometry of $T_{i}$ by universality. Hence, $t_{i}$ is a closed set. Now, let $u$ be a modular complement of $t_{i}$. The 
function from the interval $[u, \hat{1}]$ to $\left[\hat{0}, t_{i}\right]$ defined by $x \mapsto x \wedge t_{i}$ is a surjection. Since $u$ is a modular complement, $[u, \hat{1}]$ contains a copy of $T_{i}$ as a subgeometry (viz. the image of $\left[0, t_{i}\right]$ under the map $\left.x \mapsto x \vee u\right)$; as $T_{i}$ is universal, $[u, \hat{1}]$ is isomorphic to $T_{i}$. Thus, the surjection is in fact a bijection.

In particular, if $v$ strictly contains $u, v \wedge t_{i} \neq \hat{0}$. Thus, none of the flats lying above $u$ can be a complement of $t_{i}$ : that is, no two complements of $t_{i}$ are comparable. By a result of Stanley [11, Theorem 1], $t_{i}$ is modular.

An immediate consequence of the lemma is that the universal model $T_{n}$ is supersolvable. Recall that a geometric lattice of rank $n$ is supersolvable if there exists a (saturated) chain $\hat{0}=x_{0}<x_{1}<\cdots<x_{n-1}<x_{n}=\hat{1}$ with rank $x_{i}=i$ such that every flat $x_{i}$ is modular [12].

Proposition 2. The universal model $T_{n}$ is supersolvable with a chain $\hat{0}=t_{0}<t_{1}<$ $\cdots<t_{n}=\hat{1}$ of modular flats such that the subgeometry $t_{i}$ is isomorphic to $T_{i}$.

Proof. As $\mathcal{T}$ is closed under direct sum, $T_{n-1} \oplus T_{1}$ occurs as a subgeometry of $T_{n}$; in particular, there is a subset $t_{n-1}$ in $T_{n}$ isomorphic to $T_{n-1}$. This set $t_{n-1}$ is a modular copoint. We can now repeat the argument on the subgeometry $t_{n-1}$.

Another consequence is a technical tool we shall use time and again.

LEMMA 3 (THE PROJECTION ARGUMENT). Let $t_{n-1}$ be a copoint of $T_{n}$ isomorphic to $T_{n-1}$ and let $u=\left\{x_{1}, \ldots, x_{m}\right\}$ be a set of points of $T_{n}$. Let $z$ be a point of $T_{n}$ which is not in the union $t_{n-1} \cup \bar{u}$. Then the map $x_{i} \rightarrow \overline{x_{i} z} \wedge t_{n-1}$ determines an isomorphism of $u$ with a subgeometry of $t_{n-1}$.

Proof. By Lemma $1, \overline{x_{i} z} \wedge t_{n-1}$ is a point.

We abbreviate the above situation by the locution: The configuration $u$ is projected onto the copoint $t_{n-1}$ via contraction by $z$.

4. Two disconnected examples. The order of the sequence $\left(T_{n}\right)$ of universal models is the positive integer $\left|T_{2}\right|-1$. We first consider the case when $q$ equals one; that is, when $T_{2}$ is a two-point line.

Proposition 4. Let $q=1$. Then $T_{n}$ is the Boolean algebra (or free geometry) $B_{n}$ on $n$ elements.

Proof (Greene [8]). We proceed by induction on the index $n$. By hypothesis, all the copoints in $T_{n}$ are isomorphic to the Boolean algebra $B_{n-1}$ and hence all $n$-element sets are independent. Thus, $T_{n}$ is a truncated Boolean algebra. But if it were nontrivially truncated, there would exist a rank two upper minor containing a three-point line.

The Boolean algebras $B_{n}$ are the universal models of the variety of free geometries. They are also the only geometries in this variety.

We can now assume the the order $q$ is greater than one.

Lemma 5. Suppose that $T_{3}$ is connected. Then $T_{n}$ is connected for all $n$.

Proof. It suffices to show that for $n \geqslant 4$, if $T_{n-2}$ and $T_{n-1}$ are connected, then so is $T_{n}$. Suppose that $T_{n}$ is disconnected. As $T_{n-1}$ is connected and $T_{n}$ contains $T_{n-1} \oplus T_{1}$ as a subgeometry, we conclude that $T_{n} \cong T_{n-1} \oplus T_{1}$. But $T_{n}$ also contains 
$T_{n-2} \oplus T_{2}$. Let $t_{n-2}$ be the subset in $T_{n}$ isomorphic to $T_{n-2}$; then $T_{n} / t_{n-2} \cong T_{2}$. Now, $t_{n-2}$, being connected, must be contained in the summand isomorphic to $T_{n-1}$. Hence, $T_{n} / t_{n-2} \cong\left(T_{n-1} / t_{n-2}\right) \oplus T_{1} \cong T_{1} \oplus T_{1}$. This implies that $T_{2}$ is a two-point line, contrary to our standing assumption that $q \geqslant 2$.

Is there a sequence $\left(M_{n}\right)$ of universal models with $M_{3}$ disconnected? If so, we must have $M_{3} \cong M_{2} \oplus M_{1}$ where $M_{2}$ is a line with $q+1$ points. A moments thought yields: $M_{4} \cong M_{2} \oplus M_{2}$. We can now show by induction that

$$
M_{2 n+1} \cong M_{2} \oplus \cdots \oplus M_{2} \oplus M_{1} \quad \text { and } \quad M_{2 n} \cong M_{2} \oplus \cdots \oplus M_{2}
$$

(where there are $n$ copies of $M_{2}$ in each sum). By taking all subgeometries of the geometries $M_{n}$, we obtain a variety of geometries. The reader should have no difficulty in seeing why we call it the variety of matchstick geometries of order $q$.

We can now assume that the universal models $T_{n}$ are all connected.

5. Having enough points. We have arrived at the first branch (of two) in our proof. First, a definition. We say that a geometry splits if it is the union of two of its proper flats (where we regard flats as point sets).

From here until $\S 8$, we assume that no $T_{n}$ splits. This condition ensures that there are "enough points" to perform certain contractions.

LEMma 6 (UPPER HOMOGENEITY). Let $p$ be any point in $T_{n+1}$. Then there exists a copoint in $T_{n+1}$ isomorphic to $T_{n}$ and not containing $p$. In particular, the contraction $T_{n+1} / p$ is isomorphic to $T_{n}$.

Proof. The result being trivial for $n=1$, we assume that $n$ is at least 2. Let $p$ be a point in $T_{n+1}$ and consider an embedding $i_{2 n}$ of $T_{n+1}$ into $T_{2 n}$. As $T_{2 n}$ contains the direct sum $T_{n} \oplus T_{n}$ and the image of $p$ can be in at most one of the summands, there exists a flat $t_{n}$ in $T_{2 n}$ isomorphic to $T_{n}$ not containing the point $i_{2 n}(p)$. Now as $T_{2 n}$ does not split, there exists a point $z$ such that $z$ is not in the union $\left(t_{n} \vee i_{2 n}(p)\right) \cup$ $i_{2 n}\left(T_{n+1}\right)$. By our choice of $z$, the image of $i_{2 n}\left(T_{n+1}\right)$ in the contraction $T_{2 n} / z$ is isomorphic to $T_{n+1}$ and the image $t_{n}^{\prime}$ of $t_{n}$ is a flat in $T_{2 n} / z$ isomorphic to $T_{n}$ and not containing the image of $i_{2 n}(p)$. By universality, there is an embedding of $T_{2 n} / z$ into $T_{2 n-1}$. Let $i_{2 n-1}$ be the composition of the maps

$$
T_{n+1} \rightarrow T_{2 n} \rightarrow T_{2 n} / z \rightarrow T_{2 n-1}
$$

In $T_{2 n-1}$, there is a flat (the image of $t_{n}^{\prime}$ ) which is isomorphic to $T_{n}$ and does not contain $i_{2 n-1}(p)$. As $T_{2 n-1}, T_{2 n-2}, \ldots, T_{n+2}$ do not split, we can repeat this argument to obtain an isomorphism $i_{n+1}$ of $T_{n+1}$ into $T_{n+1}$ such that there is a flat $t_{n}^{\prime \prime}$ of $T_{n+1}$ isomorphic to $T_{n}$ and not containing $i_{n+1}(p)$. The inverse image $i_{n+1}^{-1}\left(t_{n}^{\prime \prime}\right)$ is a copoint of $T_{n+1}$ not containing $p$.

Now, consider the set of all copoints in $T_{n}$ isomorphic to $T_{n-1}$. By the lemma, their intersection is $\hat{0}$. Thus, there exists a set of $n$ such copoints for which the intersection is $\hat{0}$. Let $t^{1}, \ldots, t^{n}$ be such a set of copoints. As these copoints are modular, the meet sublattice they generate in $T_{n}$ is a Boolean algebra. In particular, the intersections

$$
p_{i}=t^{1} \wedge \cdots \wedge t^{i-1} \wedge t^{i+1} \wedge \cdots \wedge t^{n}
$$


are points in $T_{n}$. The subgeometry of $T_{n}$ obtained by restricting $T_{n}$ to the set of points $\cup t^{i}$ is denoted by $\left\langle p_{1}, \ldots, p_{n}\right\rangle$; it is called the frame of $T_{n}$ generated by the joints $p_{1}, \ldots, p_{n}$.

What is the span of $p_{1}, \ldots, p_{k}$ ? This span, being the intersection $t_{k+1} \wedge \cdots \wedge t_{n}$ of modular copoints, is a modular flat of rank $k$. It is in fact isomorphic to $T_{k}$. (For, by modularity, $\left[\hat{0}, \overline{p_{1} \cdots p_{k}}\right] \cong[u$, 1$]$, where $u$ is any complement of $\overline{p_{1} \cdots p_{k}}$; but $T_{n}$ is upper homogeneous.)

We now arrive at the second branch in our proof. Choose a frame in $T_{3}$. Two possibilities may occur: the frame is all of $T_{3}$; or there may be more points in $T_{3}$. The first possibility leads to Dowling geometries while the second leads to projective geometries.

6. Projective geometries. We assume in this section that $T_{3}$ contains more points than a frame. Ultimately, we shall show that this implies the order $q$ of $\left(T_{n}\right)$ is a prime power and $T_{n}$ is the projective geometry $P_{n}(q)$.

We begin with an easy fact.

Lemma 7. Let $\left(T_{n}\right)$ be of order $q$. Then

$$
\left|T_{n}\right| \leqslant 1+q+q^{2}+\cdots+q^{n-1},
$$

with equality if and only if $T_{n}$ is a projective geometry of order $q$.

Proof. For $n \geqslant 1$, let $a_{n}=\left|T_{n}\right|-\left|T_{n-1}\right|$. We prove by induction that $a_{n} \leqslant q^{n-1}$; this will clearly imply the inequality. For $n=1$ or 2 , the assertion holds trivially. Now consider $n \geqslant 3$. Let $t_{n-2}<t_{n-1}$ be a chain in $T_{n}$ consisting of a coline and a copoint isomorphic to $T_{n-2}$ and $T_{n-1}$ (respectively). There are $q$ other copoints covering $t_{n-2}$ and by induction, these copoints $u$ satisfy: $|u|-\left|t_{n-2}\right| \leqslant q^{n-2}$. Hence, $\left|T_{n}\right|-\left|T_{n-1}\right| \leqslant q \cdot q^{n-2}$.

Suppose now that equality is attained. As $T_{n}$ is connected, it suffices to show that $T_{n}$ is a modular geometry [2, p. 93]; as is easy to show, this is equivalent to showing that every line is modular, which we prove by showing that every line contains at least $q+1$ points. We proceed by induction. In $T_{1}$ and $T_{2}$, every line has $q+1$ points. Now, let $t_{n-2}$ be a coline isomorphic to $T_{n-2}$ in $T_{n}$. By the equality, every copoint lying above $t_{n-2}$ is isomorphic to $T_{n-1}$. Using the induction hypothesis, every line contained in one of these copoints has $q+1$ points. Finally, consider a line $l$ not contained in any of these copoints. This line $l$ must intersect every copoint covering $t_{n-2}$ and hence must have $q+1$ points.

Let $\left\langle p_{1} p_{2} p_{3}\right\rangle$ be a frame in $T_{3}$. By assumption, there is a point-an exterior point -in $T_{3}$ not in the frame. Consider now a frame $\left\langle p_{1} p_{2} p_{3} p_{4}\right\rangle$ in $T_{4}$. Let $z$ be an exterior point of the plane $\overline{\left\langle p_{2} p_{3} p_{4}\right\rangle}$ and let $p$ be the intersection of the lines $\overline{p_{4} z}$ and $\overline{p_{2} p_{3}}$. Contracting by the point $z$, we project the line $\overline{p_{1} p_{4}}$ onto the line $\overline{p_{1} p}$. We conclude that $\overline{p_{1} p}$ has $q+1$ points, of which $q-1$ are exterior points. Similarly, each contraction (of $T_{4}$ ) by one of these $q-1$ points projects $\overline{p_{3} p_{4}}$ onto a line containing $p_{4}$ and $q-1$ exterior points in $\overline{p_{1} p_{2} p_{4}}$. This gives at least $(q-1)^{2}$ exterior points in $\overline{p_{1} p_{2} p_{4}}$ (hence in $T_{3}$ ), which by the lemma makes $T_{3}$ a projective plane of order $q$. 
Our final step is to show that $T_{n}$ is a projective geometry. Again, we proceed by induction, having already proved the assertion for $n \leqslant 3$. Suppose that the assertion is true for $m \leqslant n-1$. Let $\left\langle p_{1} \cdots p_{n+1}\right\rangle$ be a frame in $T_{n+1}$ and let $t=\overline{p_{1} \cdots p_{n-2}}$. As observed earlier, $t$ is isomorphic to $T_{n-2}$ and is thus a projective geometry of rank $n-2$. Let $u$ be any flat covering $t$ and contained in the modular flat $\overline{p_{1} \cdots p_{n}}$. The flat $\overline{p_{1} \cdots p_{n}}$ is isomorphic to $T_{n}$. We shall show that it is in fact a projective geometry. Consider the intersection $u \wedge \overline{p_{n-1} p_{n}}$. As $\overline{p_{n-1} p_{n}}$ is a modular line, this intersection is a point $a$. Obviously, $u$ is the closure of $p_{1}, \ldots, p_{n-2}, a$. Now, in $T_{n+1}$, consider the line $\overline{a p_{n+1}}$. This line is contained in the projective plane $\overline{p_{n-1} p_{n} p_{n+1}}$ and hence there exists a point $z$ in $\overline{a p_{n+1}}$ distinct from $a$ and $p_{n+1}$. Contracting by the point $z$, we project the projective geometry (of rank $n-1) \overline{p_{1} \cdots p_{n-2} p_{n+1}}$ onto $u\left(u\right.$ being contained in $\left.\overline{p_{1} \cdots p_{n}}\right)$. We conclude that $u$ is a projective geometry of rank $n-1$; indeed, every flat contained in $\overline{p_{1} \cdots p_{n}}$ covering $t$ is a projective geometry. Counting up all the points, we obtain the equality

$$
\left|T_{n}\right|=\left|\overline{p_{1} \cdots p_{n}}\right|=1+q+\cdots+q^{n-1} .
$$

By the lemma, $T_{n}$ is a projective geometry of rank $n$. Finally, we note that, as is well known, projective geometries of order $q$ and rank higher than three exist only if $q$ is a prime power.

7. Dowling geometries. We now tackle the possibility that $T_{3}$ consists only of a frame. Our first result is that this property propagates upwards.

LEMMA 8. If $T_{3}$ consists only of a frame, then $T_{n}$ also consists only of a frame.

Proof. Let $n$ be the first index for which the lemma is false. Choose a frame $\left\langle p_{1}, \ldots, p_{n}\right\rangle$ in $T_{n}$ and let $z$ be a point in $T_{n}$ not in the frame. Consider the intersection $\overline{p_{1} \cdots p_{n-1}} \wedge \overline{p_{n} z}$. By modularity, this is a point contained in the flat $\overline{p_{1} \cdots p_{n-1}}$ (which is isomorphic to $T_{n-1}$ ), but it is not contained in the frame $\left\langle p_{1}, \ldots, p_{n-1}\right\rangle$. This is a contradiction.

Our next task is to determine the structure of $T_{3}$. Let $\left\langle p_{1} p_{2} p_{3}\right\rangle$ be a frame for $T_{3}$. There are exactly three modular lines: $l_{12}=\overline{p_{1} p_{2}}, l_{23}=\overline{p_{2} p_{3}}$ and $l_{13}=\overline{p_{1} p_{3}}$. Let $a$ be an interior point (i.e. a point distinct from the joints) of $l_{12}$, and let $b$ be any interior point of $l_{23}$. The line $\overline{a b}$ meets the modular line $l_{13}$ in a unique point $c$, and consists precisely of $a, b$ and $c$. Such lines $\overline{a b}$ are called transversals. We interpret the transversal $\{a, b, c\}$ as the equation $a b=c$ and form a multiplication table with the interior points of $l_{12}$ labelling the rows, the interior points of $l_{23}$ labelling the columns, and the interior points of $l_{13}$ as the entries. This multiplication table is a Latin square. Our next step is to show that it can be labelled so as to form the multiplication table of a group.

To do so, we move up to $T_{4}$; there are four copies of $T_{3}$ (called facets) in $T_{4}$, viz. $t_{i j k}=\overline{p_{i} p_{j} p_{k}}$ where $i, j$ and $k$ are elements from the set $\{1,2,3,4\}$. We shall always choose $i, j$ and $k$ so that $i<j<k$. Again, we denote the modular line $\overline{p_{i} p_{j}}$ by $l_{i j}$.

We now label the interior points in the six modular lines $l_{i j}$. Choose interior points $a_{12}, a_{13}$ and $a_{14}$ in the lines $l_{12}, l_{13}$ and $l_{14}$ respectively. Label these points $\varepsilon ; \varepsilon$ will turn out to be the identity element of the group. Further, label the interior 
points in $l_{12}$ (by Greek letters, say). We will make use of the following two procedures for extending the labelling.

(A) Suppose that the points $b$ on $l_{i j}$ and $a_{j k}$ on $l_{j k}$ have been labelled $\alpha$ and $\varepsilon$ respectively, and that $b^{\prime}$ is the point of $l_{i k}$ for which $\left\{b, a_{j k}, b^{\prime}\right\}$ is a transversal. Then label $b^{\prime}$ by $\alpha$.

(B) Suppose that the points $a_{i j}$ on $l_{i j}$ and $b$ on $l_{i k}$ have been labelled $\varepsilon$ and $\alpha$ respectively, and that $b^{\prime}$ is the point of $l_{j k}$ for which $\left\{a_{i j}, b^{\prime}, b\right\}$ is a transversal. Then label $b^{\prime}$ by $\alpha$.

The reader should keep in mind here that multiplication in the quasigroup corresponding to $t_{i j k}$ is given by:

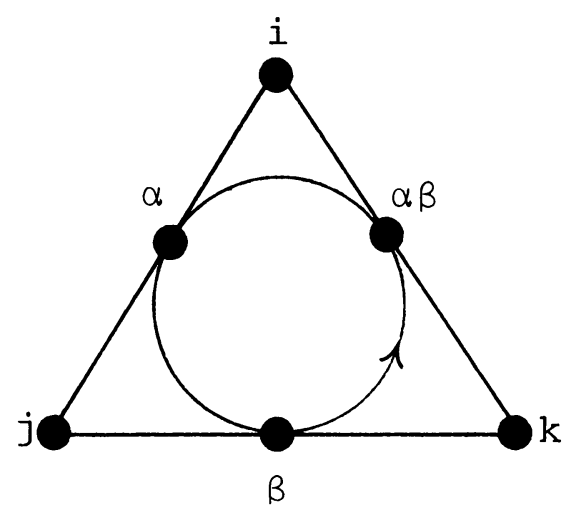

The following steps extend the labelling to all interior points of the line $l_{i j}$.

(1) Apply (B), first with $(i, j, k)=(1,2,3)$ and $b=a_{13}$, then with $(i, j, k)=$ $(1,2,4)$ and $b=a_{14}$. This gives us points labelled $\varepsilon$ on $l_{23}$ and $l_{24}$.

(2) Apply (A) with $(i, j, k)=(1,2,3)$ and $b$ ranging over all interior points of $l_{12}$ to obtain labels for the points of $l_{13}$. Repeat, replacing 3 by 4 , to obtain labels for $l_{14}$.

(3) Apply (B) with $(i, j, k)=(1,2,3)$ and $b$ ranging over the interior points of $l_{13}$ to obtain labels for $l_{23}$. Repeat, replacing 3 by 4 , to obtain labels for $l_{24}$.

(4) Apply (B) with $(i, j, k)=(1,3,4)$ and $b$ ranging over the interior points of $l_{14}$ to obtain labels for $l_{34}$.

This completes the labelling. Notice that step (4) above was one of four different ways of obtaining a labelling of $l_{34}$, the other three being application of (A) with $(i, j, k)=(1,3,4)$, and application of $(\mathrm{A})$ or $(\mathrm{B})$ with $(i, j, k)=(2,3,4)$. We will show momentarily that all of these procedures lead to the same labelling. Here, the basic observation is

Lemma 9. Let $\{a, b, c\},\{c, d, e\}$ and $\{a, f, e\}$ be transversals on three distinct facets of $T_{4}$. Then, $\{b, d, f\}$ is a transversal on the fourth facet.

Proof. Without loss of generality, we may assume that $\{a, b, c\},\{c, d, e\}$ and $\{a, f, e\}$ are transversals on the facets $t_{123}, t_{134}$ and $t_{124}$ respectively. In the contraction of $T_{4}$ by $b, a$ is projected onto $c, \overline{a e}$ onto $\overline{c e}$ and $l_{24}$ onto $l_{34}$. Therefore, $f=\overline{a e} \wedge l_{24}$ is projected onto $\overline{c e} \wedge l_{34}=d$. 
In what follows we use $\alpha_{i j}$ to denote the point of $l_{i j}$ which is labelled $\alpha$.

We may now verify that each of the three alternate procedures mentioned above produces our present labelling of $l_{34}$. We first observe that since $\left\{\varepsilon_{12}, \varepsilon_{23}, \varepsilon_{13}\right\}$, $\left\{\varepsilon_{12}, \varepsilon_{24}, \varepsilon_{14}\right\}$ and $\left\{\varepsilon_{13}, \varepsilon_{34}, \varepsilon_{14}\right\}$ are transversals, Lemma 9 says that $\left\{\varepsilon_{23}, \varepsilon_{34}, \varepsilon_{24}\right\}$ is also. But then, as $\left\{\alpha_{12}, \varepsilon_{23}, \alpha_{13}\right\}$ and $\left\{\alpha_{12}, \varepsilon_{24}, \alpha_{14}\right\}$ are also transversals, $\left\{\alpha_{13}, \varepsilon_{34}, \alpha_{14}\right\}$ must be one as well. That is, we would have obtained the same labelling of $l_{34}$ by applying (A) with $(i, j, k)=(1,3,4)$. That the remaining two procedures give the same result is shown similarly: $\left\{\alpha_{23}, \varepsilon_{34}, \alpha_{24}\right\}$ is a transversal because $\left\{\varepsilon_{12}, \alpha_{23}, \alpha_{13}\right\}$, $\left\{\alpha_{13}, \varepsilon_{34}, \alpha_{14}\right\}$ and $\left\{\varepsilon_{12}, \alpha_{24}, \alpha_{14}\right\}$ are; and $\left\{\varepsilon_{23}, \alpha_{34}, \alpha_{24}\right\}$ is a transversal because $\left\{\varepsilon_{12}, \varepsilon_{23}, \varepsilon_{13}\right\},\left\{\varepsilon_{12}, \alpha_{24}, \alpha_{14}\right\}$ and $\left\{\varepsilon_{13}, \alpha_{34}, \alpha_{14}\right\}$ are.

Now each of the four facets $t_{i j k}$ defines a multiplication as described above. We need to know that these multiplications are the same. But contraction by $a_{34}$ (which equals $\varepsilon_{34}$ ) fixes $\alpha_{12}$ and projects $\beta_{23}$ onto $\beta_{24},(\alpha \beta)_{13}$ onto $(\alpha \beta)_{14}$, so that $t_{123}$ and $t_{124}$ define the same multiplication. Similarly we can equate the multiplications of $t_{124}$ and $t_{134}$ by contracting $a_{23}$ and of $t_{134}$ and $t_{234}$ by contracting $a_{12}$. Thus all multiplications are the same.

We are now ready to show that our multiplication table is the multiplication table of a group. The only axiom requiring proof is the associative law. Consider the transversals $\left\{\alpha_{12}, \beta_{23},(\alpha \beta)_{13}\right\},\left\{(\alpha \beta)_{13}, \gamma_{34},(\alpha \beta) \gamma_{14}\right\}$, and $\left\{\beta_{23}, \gamma_{34},(\beta \gamma)_{24}\right\}$. By the lemma, $\left\{\alpha_{12},(\beta \gamma)_{24},(\alpha \beta) \gamma_{14}\right\}$ is also a transversal. Hence, $(\alpha \beta) \gamma=\alpha(\beta \gamma)$. We denote the abstract group defined by our multiplication table by $A$. As Dowling has shown (his proof of Theorem 8 in [7] applies here), the group $A$ is determined up to isomorphism by $T_{3}$.

What are the dependencies in $T_{n}$ ? The easiest way to describe them is to consider the following graphical representation. (The ideas and terminology here are based on Zaslavsky $[14,15]$.) We construct the graph $\bar{K}_{n}$ on the vertex set $\{1,2, \ldots, n\}$ by inserting between each pair of vertices $i$ and $j$ (with $i<j$ ) $|A|$ multiple edges labelled by the elements of $A$ and directed from $i$ to $j$. The graph $\bar{K}_{n}$ is thus a "flattened" representation of the geometry $T_{n}$. We identify the points of $T_{n}$ with the vertices and edges of $\bar{K}_{n}$ by: $p_{i} \mapsto i$ and $\alpha_{i j} \mapsto$ the edge labelled $\alpha$ between the vertices $i$ and $j$.

Let $S$ be the union of $\{1,2, \ldots, n\}$ and the edge set of $\bar{K}_{n}$. A subset $C \subseteq S$ is called a cycle if it consists of a single vertex or it is a cycle (which is not necessarily directed) in the graph-theoretic sense: that is, if the elements of $C$ (which are all edges) can be rearranged so that $e_{1}$ is an edge between $i_{0}$ and $i_{1}, e_{2}$ is an edge between $i_{1}$ and $i_{2}, \ldots$ and $e_{m}$ is an edge between $i_{m-1}$ and $i_{0}$, and $i_{0}, \ldots, i_{m}$ are distinct vertices. Fix a cycle $C$ of edges. Consider the label map $\lambda: C \rightarrow A$ defined on the edges of $C$ by: for $e_{j}$ labelled $\alpha, \lambda\left(e_{j}\right)=\alpha$ if $i_{j-1}<i_{j}$ and $\alpha^{-1}$ if $i_{j-1}>i_{j}$. The cycle $C$ is said to be balanced if $\lambda\left(e_{1}\right) \cdots \lambda\left(e_{m}\right)=\varepsilon$ (this condition is clearly independent of our choice of starting point); $C$ is unbalanced otherwise. We also insist that a cycle consisting of a single vertex is unbalanced; cycles of this kind are called unbalanced loops. Besides cycles, we need three other kinds of subgraphs of $\bar{K}_{n}$. A theta graph is a set of edges $\left\{e_{1}, \ldots, e_{k}, f_{1}, \ldots, f_{l}, g_{1}, \ldots, g_{m}\right\}$ such that $\left\{f_{1}, \ldots, f_{l}\right\}$ is nonempty, and

$$
\left\{e_{1}, \ldots, e_{k}, f_{1}, \ldots, f_{l}\right\}, \quad\left\{e_{1}, \ldots, e_{k}, g_{1}, \ldots, g_{m}\right\}, \quad \text { and } \quad\left\{f_{1}, \ldots, f_{l}, g_{1}, \ldots, g_{m}\right\}
$$


are cycles. A handcuff is a subset of $S$ of the form

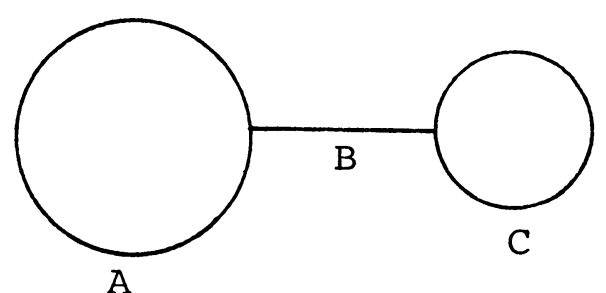

where $A$ and $C$ are cycles (thus $A$ or $C$ may consist of a single vertex) and $B$ is a simple path. In the special case that $B$ is a single vertex, we also call the cycle a figure-of-eight. A bicycle (or bicircular graph) is a theta graph or a handcuff.

There are four kinds of circuits in the plane $t_{i j k}$ (which equals the span of $p_{i}, p_{j}$ and $p_{k}$ ), namely $\left\{\alpha_{i j}, \beta_{j k},(\alpha \beta)_{i k}\right\},\left\{r, \alpha_{r s}, s\right\},\left\{r, \alpha_{r s}, \beta_{r s}\right\}$ and $\left\{\alpha_{r s}, \beta_{r s}, \gamma_{r s}\right\}$; here, in the latter three types, we use $r$ and $s$ to stand for any pair of elements from $\{i, j, k\}$. Circuits of the first type are balanced cycles; those of the remaining three types are bicycles containing no balanced cycles. We call these circuits the atomic circuits of $T_{n}$.

For brevity, we use the term Z-set for a set which is either a balanced cycle, or a bicycle none of whose cycles is balanced. Clearly, no $Z$-set properly contains another. We shall show that the $Z$-sets are all the circuits of $T_{n}$ by showing, firstly, that any subset of $S$ not containing a $Z$-set is independent, and secondly, that any $Z$-set is dependent.

Suppose that $I$ is a dependent subset of $S$ which contains no $Z$-set. We may assume that $I$ is a circuit. Decompose $I$ into its connected components (in the graph-theoretic sense). As $I$ contains no $Z$-set, any connected component of $I$ can contain at most one unbalanced cycle. Let $m$ be the maximum size of an unbalanced cycle in $I$. Consider first the case $m \geqslant 3$. Let $\left\{e_{1}, \ldots, e_{m}\right\}$ (arranged so that $e_{i-1}$ and $e_{i}$ are incident) be an unbalanced cycle in $I$ of size $m$. Take the circuit $I$ and the atomic circuit $\left\{e_{m-1}, e_{m}, f\right\}$ (where $\left\{e_{m-1}, e_{m}, f\right\}$ is a transversal). By the circuit elimination property (see e.g. [13, p. 23]) we can eliminate the edge $e_{m}$ from $I \cup\{f\}$, thus obtaining a circuit $I^{\prime}$ containing fewer unbalanced cycles of size $m$ than $I$. Using this argument repeatedly, we can reduce to the case $m \leqslant 2$. If $m=2$, let $\left\{e_{1}, e_{2}\right\}$ be an unbalanced cycle of size two in $I$. Consider the atomic circuit $\left\{e_{1}, e_{2}, i\right\}$, where $i$ is one of the endpoints of $e_{1}$ (and $e_{2}$ ). By the circuit elimination property, we can obtain a circuit $I^{\prime}$ containing one less unbalanced cycle of size two than $I$. Iterating this argument, we may assume that $I$ contains at most one unbalanced loop (which is the only possible unbalanced cycle of length one) in each connected component.

Now let $i$ be an unbalanced loop in $I$ occurring in a connected component of $I$ which has size at least two. Let $e$ be an edge of $I$ incident on $i$ and let $j$ be the other endpoint of $e$. Consider the atomic circuit $\{i, e, j\}$; by circuit elimination, we can eliminate $e$ from $I \cup\{j\}$ to obtain another circuit $I^{\prime}$ containing fewer edges than $I$. Similarly, if $e$ is an edge (with endpoints $i$ and $j$ ) in a component of $I$ containing no unbalanced loop, we can eliminate $e$ from the union of $\{i, e, j\}$ and $I$ to obtain a circuit $I$ containing fewer edges than $I$. All told, these elimination arguments allow 
us to assume that $I$ contains no edges - that is to say, $I$ is a union of unbalanced loops. But the set $\{1,2, \ldots, n\}$ of unbalanced loops is an independent set. This contradiction proves that any set which contains no $Z$-set is independent.

The proof that all $Z$-sets are dependent consists in showing that every $Z$-set may be obtained from the atomic circuits by iterating circuit elimination. The details, which are easy but tedious, are left to the reader.

We have now shown that the circuits of $T_{n}$ must be the $Z$-sets. That the $Z$-sets do indeed form the circuits of a geometry is proved in [14 and 15]. The universal model $T_{n}$ thus obtained is the Dowling geometry $Q_{n}(A)$ of rank $n$ based on the finite group $A$ (see [7]). The variety specified by these universal models is called the variety of voltage-graphic geometries with voltages in $A$. (Our description of $Q_{n}(A)$ differs somewhat from that of [7]. For a proof of the equivalence when $A$ is cyclic-which should indicate the proof in the general case-see [ 5 or 6].)

8. The split case: origami geometries. We return now to the first branch of the proof. Assume that some $T_{m}$ splits and choose $n$ to be the least integer for which $T_{n+1}$ splits. Since $T_{2}$ is connected it does not split, and we may assume that $n$ is at least two. We shall in fact show that $n$ must be equal to two.

Lemma 10. Suppose $T \in \mathcal{T}$ contains flats $t_{m}$ and $t_{r}$ isomorphic to $T_{m}$ and $T_{r}$ respectively and that the rank of their intersection $t_{m} \wedge t_{r}$ is $k$. Then if $r \leqslant n, t_{m} \wedge t_{r}$ is isomorphic to $T_{k}$.

Proof. We induct on $r$, the (smallest) case $r=k$ being trivial. Let $t_{m} \wedge t_{r}=t$.

Let $t_{r-1}$ be a flat in $t_{r}$ isomorphic to $T_{r-1}$, and let $x$ be a point of $t_{r}$ not in $t_{r-1} \cup t$. (Such a point exists since $t_{r}$ does not split.) If we let $t_{m}^{\prime}$ and $t_{r-1}^{\prime}$ be the images of $t_{m}$ and $t_{r}$ under contraction by $x$, then $t_{m}^{\prime}$ and $t_{r-1}^{\prime}$ are flats of $T / x$ isomorphic to $T_{m}$ and $T_{r-1}$ respectively. Moreover, $t_{m}^{\prime} \wedge t_{r-1}^{\prime}$ is isomorphic to $t$ (since it is the image of $t$ ), and to $T_{k}$ (by the induction hypothesis). Thus $t$ is isomorphic to $T_{k}$.

We must investigate in some detail the structure of the geometries $T_{m}$. A first step in this direction is

Lemma 11. For each $m=n+1, \ldots, 2 n$, there exist flats $t$ and $t^{\prime}$ in $T_{m}$, isomorphic to $T_{n}$, such that $t \vee t^{\prime}=T_{m}$.

Proof. The result is true for $m=2 n$ as $T_{2 n}$ contains the direct sum $T_{n} \oplus T_{n}$. Moreover, if it is true for $m+1$ and there exists a point $x$ in $T_{m+1}$ but not in $t \cup t^{\prime}$, then contraction by $x$ gives the result for $m$.

So if the lemma is false, there must exist an $m_{0}(>n)$ such that the lemma holds for $m_{0}+1, \ldots, 2 n$, and $T_{m_{0}+1}=t \cup t^{\prime}$.

By connectivity $m_{0} \leqslant 2 n-2$. By Lemma 1 , we may regard $T_{m_{0}+1}$ as a flat of $T_{m_{0}+2}$. Since the lemma is true for $m_{0}+2$, there is a flat $t_{n}$ of $T_{m_{0}+2}$ which is isomorphic to $T_{n}$ and not contained in $T_{m_{0}+1}$. By Lemmas 1 and $10, t_{n-1}=t_{n} \wedge T_{m_{0}+1}$ is isomorphic to $T_{n-1}$. Since $t_{n-1}$ is contained in $t \cup t^{\prime}$ and does not split, we may assume that $t_{n-1}$ is contained in $t$. This proves the lemma for $m=n+1$, since the flat $t \vee t_{n}$ is of rank $n+1$, and by universality is a subgeometry of $T_{n+1}$. 
Finally, the validity of the lemma for $m=n+1$ rules out the existence of an $m_{0}$ as described above. For if $u, v, w, x$ are isomorphic to $T_{n}$ with $u \vee v=T_{n+1}$ and $w \vee x=T_{m_{0}+1}\left(m_{0}>n\right)$, then we may regard $T_{n+1}$ as a flat of $T_{m_{0}+1}$, and it is easily seen (because $\operatorname{rank}(u \wedge v)>\operatorname{rank}(w \wedge x))$ that $u$ and $v$ cannot both be contained in $w \cup x$.

We see in particular that $T_{n+1}$ is the union of two flats isomorphic to $T_{n}$. In fact we can show that for each $m \geqslant n, T_{m+1}$ is the union of a copy of $T_{m}$ and a copy of $T_{n}$, and can obtain a fairly precise description of how these additional copies of $T_{n}$ must be attached.

LEMMA 12. Let $m \geqslant n$.

(a) There exist flats $t_{m}$ and $t_{n}$ in $T_{m+1}$ with $t_{m}$ isomorphic to $T_{m}, t_{n}$ isomorphic to $T_{n}$, and $T_{m+1}=t_{m} \cup t_{n}$.

(b) Let $t_{n} \subseteq t_{n+1} \subseteq \cdots \subseteq t_{m}$ with $t_{i}$ isomorphic to $T_{i}$, and for $n \leqslant r \leqslant m$ let $t_{r}=\cup_{i=1}^{r-n+1} t^{i}$ with $t^{i}$ isomorphic to $T_{n}$. (This assumption is justified by (a).) If $k \leqslant n$, and $t$ is a flat of $t_{m}$ isomorphic to $T_{k}$, then $t \subseteq t^{i}$ for some $i \in\{1, \ldots, m-n+1\}$. In particular, $t^{1}, \ldots, t^{m-n+1}$ are the only copies of $T_{n}$ in $t_{m}$.

Proof. (a) By Lemma 11 there is a collection of copies of $T_{n}$ which span $T_{m+1}$. (For $m \geqslant 2 n$ this follows from $T_{m+1} \supseteq T_{m-n+1} \oplus T_{n}$.) Thus, given a copy $t_{m}$ of $T_{m}$ in $T_{m+1}$, there exists a flat $t_{n}$ of $T_{m+1}$ isomorphic to $T_{n}$ and not contained in $t_{m}$.

By Lemma $10, t_{m} \wedge t_{n}$ is isomorphic to $T_{n-1}$.

Suppose there exists a point $x$ in $T_{m+1}$ not in $t_{m} \cup t_{n}$. If $t=t_{m} \wedge\left(t_{n} \vee x\right)$, then contraction by $x$ projects $t_{n}$ onto $t$, and shows that $t$ is isomorphic to $T_{n}$. Now it is easy to show (since $T_{n}$ does not split) that if $T_{n+1}=t^{\prime} \cup t^{\prime \prime}$ with $t^{\prime}, t^{\prime \prime}$ isomorphic to $T_{n}$, then $T_{n+1}$ contains no further copies of $T_{n}$. On the other hand, the flat $t_{n} \vee x$ is a subgeometry of $T_{n+1}$ (by universality) and contains the copies $t_{n}$ and $t$ of $T_{n}$, plus the point $x$ outside $t_{n} \cup t$. This is a contradiction and we have proved (a).

(b) Let $r$ be the least index for which $t \subseteq t_{r}$. By (a), $t=\left(t \cap t^{r-n+1}\right) \cup\left(t \cap t_{r-1}\right)$. Since $t \nsubseteq t_{r-1}$ and $t$ does not split, we must have $t \subseteq t^{r-n+1}$.

Let $m \geqslant n$. By Lemma 12(b), there are exactly $m-n+1$ copies of $T_{n}$ in $T_{m}$. We take these to be the vertices of a graph $G_{m}$ in which vertices $t$ and $t^{\prime}$ are joined if and only if $t \wedge t^{\prime}$ is isomorphic to $T_{n-1}$. We will speak of $t$ both as a vertex of $G_{m}$ and as a flat of $T_{m}$. We remark that $G_{m}$ is connected by Lemma 12 .

LEMMA 13. $G_{m}$ is a path. Moreover, if we take $t^{0}, \ldots, t^{m-n}$ to be one of the (two) natural orderings of its vertices, then $\operatorname{rank}\left(t^{i} \wedge t^{j}\right)=\max \{n-j+i, 0\}$ for all $0 \leqslant i$ $\leqslant j \leqslant m$.

Proof. Let $V G_{m}$ denote the set of vertices of $G_{m}$. We first remark that for any $t, t^{\prime}$, $t^{\prime \prime} \in V G_{m}$,

$$
\begin{aligned}
n+\operatorname{rank}\left(t \wedge t^{\prime}\right) & \leqslant \operatorname{rank}\left[\left(t \wedge t^{\prime \prime}\right) \vee\left(t^{\prime} \wedge t^{\prime \prime}\right)\right]+\operatorname{rank}\left(t \wedge t^{\prime} \wedge t^{\prime \prime}\right) \\
& =\operatorname{rank}\left(t \wedge t^{\prime \prime}\right)+\operatorname{rank}\left(t^{\prime} \wedge t^{\prime \prime}\right)
\end{aligned}
$$

(The equality of the last two expressions follows from Lemmas 10 and 1.) 
The length of a path between two vertices is the number of edges in the path, and the distance, $d\left(t, t^{\prime}\right)$, between two vertices $t$ and $t^{\prime}$ is the length of a shortest path joining them. An easy consequence of $(*)$ is:

(**) If $P$ is a path of length $d$ joining $t$ and $t^{\prime}$, then $\operatorname{rank}\left(t \wedge t^{\prime}\right) \geqslant n-d$. Moreover, if $t^{\prime \prime}$ is a vertex in $P$ such that the segment of $P$ from $t$ to $t^{\prime \prime}$ has length $c$, and if $\operatorname{rank}\left(t \wedge t^{\prime \prime}\right)>n-c$, then $\operatorname{rank}\left(t \wedge t^{\prime}\right)>n-d$.

We first prove the lemma for $m=2 n$. As $T_{2 n}$ contains $T_{n} \oplus T_{n}$, there exist $t$ and $t^{\prime}$ in $V G_{2 n}$ with $t \wedge t^{\prime}=\hat{0}$. By $(* *), d\left(t, t^{\prime}\right) \geqslant n$. But $G_{2 n}$ has only $n+1$ vertices, so it must be a path from $t$ to $t^{\prime}$. Moreover, if this path has vertex sequence $t=$ $t^{0}, t^{1}, \ldots, t^{n}=t^{\prime}$, then $(* *)$ implies $\operatorname{rank}\left(t^{i} \wedge t^{j}\right)=n-j+i$ for $0 \leqslant i \leqslant j \leqslant 2 n$. This proves the lemma for $m=2 n$.

For $m<2 n$, we regard $T_{m}$ as a flat of $T_{2 n}$. Then $G_{m}$ is a connected subgraph of $G_{2 n}$ and the result follows.

For $m>2 n$, we proceed by induction. If $t^{1}, \ldots, t^{m-n}$ are the vertices of a connected subgraph of $G_{m}$, then their span (in $T_{m}$ ) is isomorphic to $T_{m-1}$. (Induction on distance shows that the rank of the span is at most $m-1$; on the other hand, Lemma 12(b) shows that their union cannot be contained in a proper subgeometry of $T_{m}$..) Thus (by induction) every connected proper subgraph of $G_{m}$ is a path, so that $G_{m}$ is either a path or a cycle. But if $G_{m}$ is a cycle, say $t^{0}, \ldots, t^{m-n}$, then our inductive hypothesis applied to the paths $t^{1}, \ldots, t^{m-n}$ and $t^{m-n}, t^{0}, t^{1}$ gives $0=$ $\operatorname{rank}\left(t^{1} \wedge t^{m n^{n}}\right)=n-2$, a contradiction. It follows that $G_{m}$ is a path, say $t^{0}, \ldots, t^{m-n}$. Since each of the (two) paths of length $m-1$ in $G_{m}$ is the graph of a flat of $T_{m}$ isomorphic to $T_{m-1}$, we also obtain the equality

$$
\operatorname{rank}\left(t^{i} \wedge t^{j}\right)=\max \{n-j+i, 0\}
$$

by induction for all pairs $(i, j)$ other than $(0, m)$. To see the equality in the outstanding case, let $t_{m-1} \subseteq T_{m}$ be the flat spanned by $t^{1}, \ldots, t^{m-n}$. Then $t^{0} \wedge t_{m-1}$ $=t^{0} \wedge t^{1}$, so that $t^{0} \wedge t^{m-n} \subseteq t^{1} \wedge t^{m-n}=\hat{0}$.

We are now in a position to prove that $n$ equals two. Before proceeding, we observe that for $n=2$, we do obtain a variety. Define the geometries $O_{m}(q)$ recursively by: $O_{2}(q)$ is a line with $q+1$ points; $O_{m}(q)$ is the union of a copy $t_{m-1}$ of $O_{m-1}(q)$ and a copy $t_{2}$ of the line $O_{2}(q)$ taken in such a way that the intersection $t_{m-1} \wedge t_{2}$ is a point and $t_{m-1}$ contains a copy of $O_{m-2}(q)$ disjoint from $t_{2}$. It is easy to deduce from Lemmas 12 and 13 that this sequence is the only sequence of universal models for which $\left|T_{2}\right|=q+1$ and $T_{3}$ splits.

Another way to describe $O_{n}(q)$ is to take the Boolean algebra on the point set $\{1,2, \ldots, n\}$. On each of the lines $\overline{12}, \overline{23}, \ldots, \overline{i(i+1)}, \ldots, \overline{(n-1) n}$, add $q-1$ points in general position. The resulting geometry is the geometry $O_{n}(q)$ defined above. The geometries $O_{n}(q)$ are called the full origami geometries of order $q$. Their subgeometries form a variety called the variety of origami geometries of order $q$. Note that the variety of free geometries is just the variety of origami geometries of order one.

It remains to show that $n$ cannot be greater than two. First we fix some notation. Let $m=\left|T_{n-1}\right|+2 n-1$ and let $T_{m}=t^{0} \cup \cdots \cup t^{m-n}$ (notation as in Lemma 13). Further, for $1 \leqslant i \leqslant m-n$, let $t_{n-1}^{i}=t^{i-1} \cap t^{i}$; for $1 \leqslant i \leqslant m-n-1$, let $t_{n-2}^{i}=$ $t^{i-1} \cap t^{i} \cap t^{i+1}$. Note that $t_{n-1}^{i}$ is isomorphic to $T_{n-1}$ and $t_{n-2}^{i}$ is isomorphic to $T_{n-2}$. 
Let us call a point $x$ of $t_{n-1}^{i}$ an exceptionable point if

(e) there is a (possibly empty) set of points (called a set of foci for $x$ ) $\left\{z_{1}, \ldots, z_{k}\right\}$ in $T_{m} \backslash \cup_{j=i}^{m-n} t^{j}$ such that there are $n$ (distinct) copies of $T_{n}$ containing the image of $x$ in the contraction $T_{m} /\left\{z_{1}, \ldots, z_{k}\right\}$.

Note that, in any geometry $T$ in the variety $\mathcal{T}$, no point can be on more than $n$ distinct copies of $T_{n}$. This follows from the fact that, by Lemma 13, any set of $n$ copies of $T_{n}$ having nonempty intersection must be of the form $\left\{t^{i}, \ldots, t^{i+n-1}\right\}$. For the same reason, a point $x$ is on $n$ copies of $T_{n}$ in $T_{m}$ itself if and only if it is the unique point in the intersection of the flats $t^{i}, \ldots, t^{i+n-1}$ for some $i$ between 0 and $m-2 n+1$. We call such exceptionable points $x$ n-points.

Assume that $n \geqslant 3$. We shall show that

(f) for $1 \leqslant i \leqslant m-2 n+1$, the number of exceptionable points on $t_{n-1}^{i+1}$ is strictly greater than the number of exceptionable points on $t_{n-1}^{i}$.

Of course, (f) leads to a contradiction: since $t_{n-1}^{1}$ contains an exceptionable point (the intersection of $\left.t^{0}, \ldots, t^{n-1}\right), t_{n-1}^{m-2 n+2}$ contains at least $m-2 n+2$ exceptionable points. But by our choice of $m, t_{n-1}^{m-2 n+2}$ contains only $m-2 n+1\left(=\left|T_{n-1}\right|\right)$ points of any kind.

Let, then, $1 \leqslant i \leqslant m-2 n+1$. Observe that there is a unique $n$-point, $x_{i+1}$ say, which is in $t_{n-1}^{i+1}$ but not in $t_{n-2}^{i}$. This point is the intersection of $t^{i}, \ldots, t^{i+n-1}$. We will have proved (f) if we can construct an injection from the set of exceptionable points in $t_{n-1}^{i} \backslash t_{n-2}^{i}$ to the set of exceptionable points which are not $n$-points in $t_{n-1}^{i+1} \backslash\left(t_{n-2}^{i} \cup\left\{x_{i+1}\right\}\right)$. To this end, choose a point $z$ in $t^{i} \backslash\left(t_{n-1}^{i} \cup t_{n-1}^{i+1}\right)$. Such a point exists since $T_{n}$ does not split. Consider the mapping $x \mapsto x:=(x \vee z) \wedge t_{n-1}^{i+1}$; that is, $x$ is mapped onto the intersection $x^{\prime}$ of the line $\overline{x z}$ and the modular flat $t_{n-1}^{i+1}$. This map is clearly an injection from $t_{n-1}^{i} \backslash t_{n-2}^{i}$ into $t_{n-1}^{i+1} \backslash t_{n-2}^{i}$. Let $x$ be an exceptionable point in $t_{n-1}^{i} \backslash t_{n-2}^{i}$ and suppose that $x^{\prime}$ is on $r$ copies of $T_{n}$ : these must be the flats $t^{i}, \ldots, t^{i+r-1}$. Suppose that $\left\{z_{1}, \ldots, z_{k}\right\}$ is a set of foci for $x$. Then, in the contraction $T_{m} /\left\{z_{1}, \ldots, z_{k}, z\right\}$, the image of $x^{\prime}$ (which is also the image of $x$ ) is on $n+r-2$ copies of $T_{n}$. (For the image of $x$ in $T_{m} /\left\{z_{1}, \ldots, z_{k}\right\}$ is on $n$ copies of $T_{n}$ and the image of $x^{\prime}$ in $T_{m} /\left\{z_{1}, \ldots, z_{k}\right\}$ is still on $r$ copies of $T_{n}$-remember that the foci are points in $T_{m} \backslash \cup_{j=i}^{m-n} t^{j}$-and contraction by $z$ destroys one copy each from the two collections of copies of $T_{n}$.) Since $x^{\prime}$ is in the intersection $t^{i} \wedge t^{i+1}, r \geqslant 2$. Hence, as $n+r-2 \leqslant n, r=2$. This implies that $x^{\prime}$ is indeed an exceptionable point (with a set of foci $\left\{z_{1}, \ldots, z_{k}, z\right\}$ ) and that $x^{\prime}$ is not an $n$-point (in particular, $\left.x^{\prime} \neq x_{i+1}\right)$. Thus, $x \mapsto x^{\prime}$ is an injection satisfying the required properties and we have proved (f). This completes the proof that $n=2$.

9. The main theorem. We have now proved the following theorem.

THEOREM 14. Let $\mathcal{T}$ be a variety of geometries with a sequence of universal models $\left(T_{n}\right)$. Then $\mathcal{T}$ is one of the following collections:
A. the variety of free geometries,
B. the variety of matchstick geometries of order $q(\$ 4)$,
C. the variety of origami geometries of order $q(\$ 8)$,
D. the variety of geometries coordinatizable over the finite field $G F(q)$, 
E. the variety of voltage-graphic geometries with voltages in a fixed finite group $A$ $(\S 7)$.

The corresponding universal models $T_{n}$ are

A. the Boolean algebra $B_{n}$,

B. the full matchstick geometry $M_{n}(q)$ of order $q$,

C. the full origami geometry $O_{n}(q)$ of order $q$,

D. the projective geometry $P_{n}(q)$ over the finite field $G F(q)$,

E. the Dowling geometry $Q_{n}(A)$ based on the finite group $A$.

One can easily deduce further results from the theorem. For example, the only varieties of binary geometries are the free geometries, the matchstick and origami geometries of order two, the graphic geometries and all binary geometries.

A more interesting result which seems difficult to prove independently is

COROLLARY 15. The only varieties which are closed under orthogonal duality are the varieties of free geometries and the varieties of geometries coordinatizable over a fixed finite field.

The proof consists of checking that the other varieties are not closed under orthogonal duality. This is obvious for the matchstick and origami geometries (with $q>1$ ). For voltage-graphic geometries, it is easy to show by a counting argument that the orthogonal dual of $Q_{4}(A)$ cannot be an $A$-labelled voltage-graphic geometry.

Our theorem says that in some sense the only nondegenerate examples of well-structured hereditary classes are the geometries coordinatizable over a finite field and voltage-graphic geometries. While the study of projective geometry over a finite field is a classical subject, the study of voltage-graphic geometries has just been initiated by Zaslavsky in [14 and 15]. Our present work would have been unimaginably more difficult had voltage-graphic geometries not been discovered.

Voltage-graphic geometries, like the coordinatizable geometries, can be described in an "economical" fashion. More precisely, to describe a voltage-graphic geometry, one need only specify a multigraph whose edges are labelled with the group elements, just as to describe a coordinatizable geometry, one need only specify a finite set of $n$-tuples of field elements. These descriptions are in general more compact (require less storage space in a computer) and easier to manipulate than purely set-theoretic descriptions such as a listing of all the circuits or bases. Thus, an important practical consequence of our theorem is that geometries belonging to a variety have compact descriptions.

What happens if we drop the restriction that our geometries be finite? Two unpleasant phenomena arise. The first is that (as we have defined it) a sequence of universal models need not be unique. For example, if $k$ is a field which is isomorphic to a subfield of itself, the projective geometry $P_{n}(k)$ is embeddable in the affine geometry $A_{n}(k)$. The second, even harder to deal with, is that there are other varieties of geometries. Apart from the existence of varieties of geometries related to the origami geometries, we also encounter varieties of algebraic geometries as in [9]; these are geometries in which the dependence relation is given by algebraic dependence over a field. Over fields of positive characteristic, it is known [13, p. 185] that 
these varieties are different from the varieties of coordinatizable geometries. Moreover, the natural universal models are not even supersolvable.

We conclude with a question:

Can varieties of finite combinatorial geometries be characterised by a finite list of excluded minors?

That is to say, for any variety $\mathcal{T}$ of geometries, does there exist a finite number of geometries $E_{1}, \ldots, E_{n}$ such that $G$ is in the variety $\mathcal{T}$ if and only if none of the geometries $E_{1}, \ldots, E_{n}$ occurs as a minor of $G$ ?

ACKNOWLEDGEMENT. The authors would like to thank the referee for his careful reading of the manuscript and many helpful suggestions.

\section{REFERENCES}

1. G. Birkhoff, On the structure of abstract algebras, Proc. Cambridge Philos. Soc. 31 (1935), 433-454.

2. __ Lattice theory, 3rd ed., Amer. Math. Soc. Colloq. Publ., vol. 25, Amer. Math. Soc., Providence, R.I., 1967.

3. P. M. Cohn, Universal algebra, Harper and Row, New York, 1965.

4. H. H. Crapo and G.-C. Rota, On the foundations of combinatorial theory: Combinatorial geometries (preliminary edition), M.I.T. Press, Cambridge, 1970.

5. P. Doubilet, G.-C. Rota and R. Stanley, On the foundations of combinatorial theory. VI: The idea of generating function, Sixth Berkeley Sympos. on Math. Statist. and Prob., Vol. II: Probability Theory, Univ. California, Berkeley, Calif., 1972, pp. 267-318.

6. T. A. Dowling, A q-analog of the partition lattice, A Survey of Combinatorial Theory (J. N. Srivastava, editor), North-Holland, Amsterdam, 1973, pp. 101-115.

7. A A class of geometric lattices hased on finite groups, J. Combin. Theory Ser. B 14 (1973), $61-86$.

8: C. Greene, Lectures in combinatorial geometries, Notes from the NSF Seminar in Combinatorial Theory, Bowdoin College, 1971, unpublished.

9. S. Mac Lane, A lattice formulation for transcendence degrees and p-bases, Duke Math. J. 4 (1938), $455-468$.

10. D. Kelly and G.-C. Rota, Some problems in combinatorial geometry, A Survey of Combinatorial Theory (J. N. Srivastava, editor), North-Holland, Amsterdam, 1973, pp. 309-312.

11. R. P. Stanley, Modular elements of geometric lattices, Algebra Universalis 1 (1971), 214-217.

12. , Supersolvable lattices, Algebra Universalis 2 (1972), 197-217.

13. D. J. A. Welsh, Matroid theory, Academic Press, London, 1976.

14. T. Zaslavsky, Biased graphs, preprint, 1977.

15. __ Signed graphs, Discrete Appl. Math. 4 (1982), 47-74.

Department of Mathematics, Massachusetts Institute of Technology, Cambridge, MasSACHUSETTS 02139

Department of Mathematics, North Texas State University, Denton, Texas 76203 